\title{
Cation hydration in hydrogelic polyacrylamide-phosphoric acid network: A study by Raman spectroscopy
}

\author{
A.M. Amorim da Costa*, A.M. Amado \\ Department of Chemistry, University of Coimbra, 3000 Coimbra, Portugal
}

Received 25 September 2000; received in revised form 15 November 2000; accepted 11 December 2000

\begin{abstract}
The effects upon the structure and morphology of adding lithium, calcium and magnesium chlorides to a phosphoric acid/polyacrylamide 2:1 molar ratio proton conducting hydrogel are examined by observing the changes in the vibrational features of the polyacrylamide chain, in the phosphate group and in the interstitial water molecules as a function of the concentration and the cationic nature of the additive, at $295 \mathrm{~K}$. On adding $\mathrm{H}_{3} \mathrm{PO}_{4}$ to the polyacrylamide hydrogel matrix, the amide groups become more accessible and the polyacrylamide-phosphoric acid network behaves like structure-makers promoting a larger association in the 'bulk' liquid water molecules. (c) 2001 Elsevier Science B.V. All rights reserved.
\end{abstract}

Keywords: Cation hydration; Polyacrylamide-phosphoric acid network; Raman spectra

\section{Introduction}

One method of producing proton conducting polymeric electrolytes is to entrap aqueous solutions of strong acids with polymeric gels [1]. In particular, it has been determined that polyacrylamide based hydrogels after doping with $\mathrm{H}_{3} \mathrm{PO}_{4}$ exhibit room temperature conductivities in the range $10^{-3}-10^{-2} \mathrm{~S}$ $\mathrm{cm}^{-1}$. In this case, the ionic conductivity of the hydrogel has been observed to increase with the increase in water concentration up to about 30-35 mass\%, remaining almost constant for higher water concentrations. In this conductivity plateau, the ionic conductivity increases with the phosphoric acid/ acrylamide molar ratio with a maximum for 1.8-2.0 mol of $\mathrm{H}_{3} \mathrm{PO}_{4}$ to $1 \mathrm{~mol}$ of acrylamide [2].

\footnotetext{
* Corresponding author. Tel./fax: +351-239-82-65-41.

E-mail address: acosta@ci.uc.pt (A.M. Amorim da Costa).
}

The electrolyte characteristics strongly depend upon the structure and morphology of the host matrixes of the prepared hydrogels. The electrical conductivity change is most likely related to the changes in the local viscosity due to $\mathrm{H}_{3} \mathrm{PO}_{4}$ interactions with both the solvent and the polymer matrix which stiffen the hydrogel [3]. High polar organic or inorganic filler additives are known to lower the polymer host crystallinity and flexibility [4,5].

Recently, in the case of composite polymers with alkali metal salts, the observed changes have been explained as resulting from acid/base interactions involving either the metal cations or the anions with the acid/base centers of the polymer [3]. The strength of the interactions between the added salts in polymer electrolytes and the polymer host, in comparison with that of the interionic forces, is currently one of the serious problems with current theories on this kind of system [6]. 
Using lithium, calcium and magnesium chlorides dissolved in a phosphoric acid/polyacrylamide 2:1 molar ratio matrix hydrogel, the present work examines the effects of the addition of these chlorides by observing the changes in the vibrational features of the polyacrylamide chain, in the phosphate group and in the interstitial water molecules as a function of the concentration and the cationic nature of the additive, at $295 \mathrm{~K}$.

\section{Experimental}

Phosphoric acid/polyacrylamide 2:1 molar ratio matrix hydrogels were prepared according to the procedure described by Wieczorek et al. [2]. Stoichiometric mixtures were prepared using reagent grade acrylamide (Merck), $N, N^{\prime}$-methylene-bis-acrylamide (Sigma), agar-agar (Montplet and Esteban) and $85 \%$ orthophosphoric acid pro analysis (Merck). Stoichiometric amounts of anhydrous lithium chloride (Sigma-Aldrich), anhydrous magnesium chloride (Sigma-Aldrich) or anhydrous calcium chloride (Merck) were added to each mixture before dropwise introduction of hydrogen peroxide to initiate the polymerization reaction. The amount of water in- cluded in each sample after the drying process was calculated on the basis of gravimetric measurements and was observed to be ca. $24 \%$.

The Raman spectra were recorded on a Ramalog double spectrometer, $0.85 \mathrm{~m}$, f/7.8, Spex model 1403, equipped with holographic gratings with 1800 grooves $/ \mathrm{mm}$. The light source used as Raman excitation was the 514.5-nm line of an argon ion laser (Coherent, model Innova 90), with ca. $50 \mathrm{~mW}$ at the sample position. The instrumental resolution was limited to $1 \mathrm{~cm}^{-1}$. A detector assembly containing a Hamamatsu type R928 photomultiplier tube, for 190-860 nm, multi alkali, in thermoelectric cooled housing, was used for detection. Raman data were stored on disk, with the possibility of further processing. The error in wavenumbers is estimated to be within ca. $1 \mathrm{~cm}^{-1}$.

\section{Results and discussion}

\subsection{The Raman vibrational spectrum of 1:2 molar} ratio polyacrylamide-phosphoric acid hydrogel

Fig. 1 shows the $140-1800 \mathrm{~cm}^{-1}$ and $2800-3700$ $\mathrm{cm}^{-1}$ Raman spectral regions, at $295 \mathrm{~K}$, for a polyacrylamide hydrogel matrix containing ca. $24 \%$ wa-

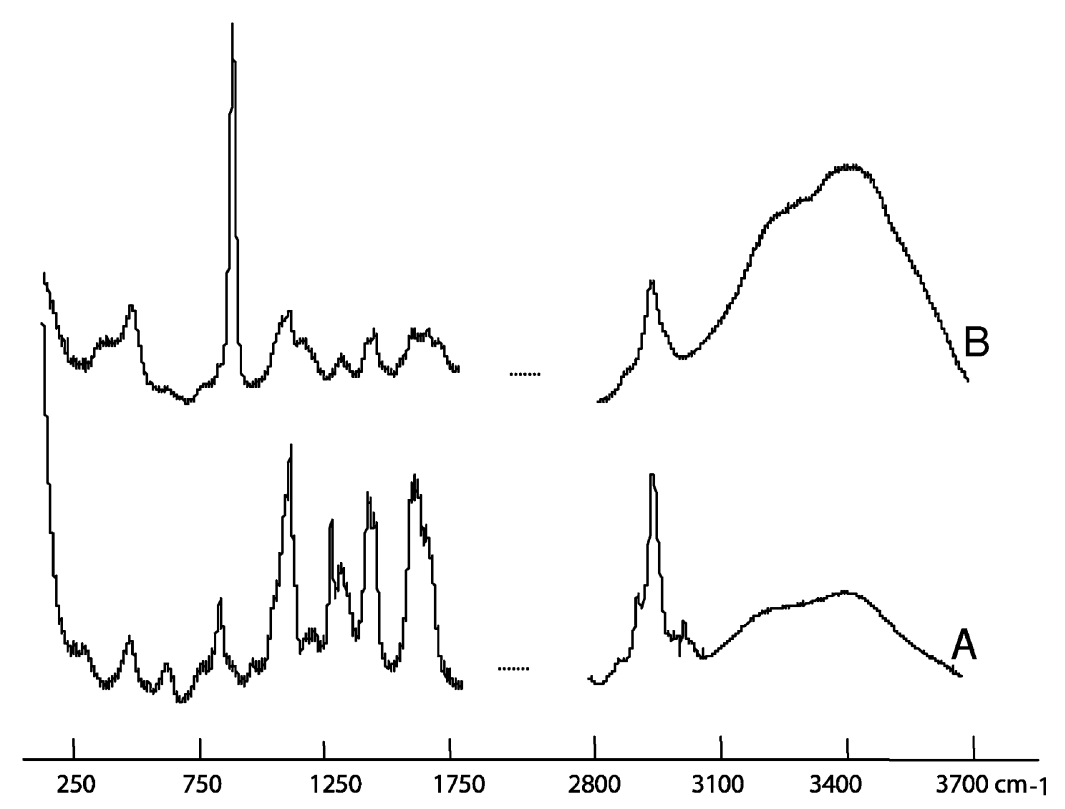

Fig. 1. Raman spectra, at $295 \mathrm{~K}$, for: (A) polyacrylamide hydrogel; (B) phosphoric acid/polyacrylamide 2:1 mole ratio hydrogel. 
ter and a 1:2 molar ratio polyacrylamide-phosphoric acid network hydrogel with the same content of water. The spectra in the first region are dominated by the internal vibrations of the polymeric acrylamide chains and the $\mathrm{H}_{3} \mathrm{PO}_{4}$ molecule. In the second region, we find the polyacrylamide $\mathrm{C}-\mathrm{H}$ stretchings $\left(2800-3000 \mathrm{~cm}^{-1}\right)$ and the broad peaks due to the amide $\mathrm{N}-\mathrm{H}$ and the phosphoric and water $\mathrm{O}-\mathrm{H}$ stretching modes $[8,9]$.

In the frequency region from 140 to $1800 \mathrm{~cm}^{-1}$, most of the vibrational modes involving the presence of $\mathrm{H}_{3} \mathrm{PO}_{4}$ are overlapped by the vibrational bands of acrylamide, except for the strong band at $895 \mathrm{~cm}^{-1}$ due to the $\mathrm{P}-\mathrm{O}(\mathrm{H})$ stretching mode. In particular, the $\mathrm{P}=\mathrm{O}$ stretching mode expected around $1250 \mathrm{~cm}^{-1}$ is overlapped by the vibrational bands due to the $\mathrm{O}-\mathrm{H}$ deformations and the $\mathrm{C}-\mathrm{C}$ stretchings [7].

For pure $\mathrm{H}_{3} \mathrm{PO}_{4}$, the frequency of this mode is observed at ca. $1001 \mathrm{~cm}^{-1}$ [1]. From this, we can anticipate a large frequency shift to a lower frequency after complexation with the polymer chains. Like the $\mathrm{P}=\mathrm{O}$ vibration, the $\mathrm{P}-\mathrm{O}(\mathrm{H})$ vibration is known to be very sensitive either to the inductive effects of substituent groups attached to the phosphorous atom or to the molecular interactions [7].

In this vibrational region, the most striking changes in the vibrations of polyacrylamide vibrations appear on the observed bands originating from the vibration of amide III and the bending mode of $\mathrm{CH}_{2}$ and on the bands originating from the vibration of amide I. At 1430 and $1455 \mathrm{~cm}^{-1}$, respectively, are the two bands originating from the $\mathrm{C}-\mathrm{N}$ stretching and the $\mathrm{CH}_{2}$ bending modes in amide III $[8,9]$. Under interactions of added salts with polyacrylamide hydrogels, the frequency of these bands remains unaffected, but there is a great increase in the intensity of the bending mode relative to the intensity of the $\mathrm{C}-\mathrm{N}$ stretching mode. This increase may be caused by the conformational changes in the polyacrylamide chain [9]. From a polyacrylamide hydrogel matrix to a polyacrylamide/lithium perchlorate 1:2 hydrogel composite, the relative intensity increase goes from $24 \%$ to ca. $32 \%$ [9]. Under the $\mathrm{H}_{3} \mathrm{PO}_{4}$ complexation with the polymer acrylamide chains, the observed change in the relative intensity of the two bands is much greater, and the $\mathrm{C}-\mathrm{N}$ stretching mode becomes less intense than the $\mathrm{CH}_{2}$ bending.
Regarding the features of polyacrylamide spectra of amide $\mathrm{I}$ in the region from 1530 to $1730 \mathrm{~cm}^{-1}$, corresponding clearly to the $\mathrm{C}=\mathrm{O}$ stretching [10], on the $\mathrm{H}_{3} \mathrm{PO}_{4}$ complexation with the polymer chains, there is an increase in the frequency of all the three components of the band envelope, as shown in Fig. 2. The components which correspond to the stretching mode of the carbonyl group of self-associated amide molecules at 1597 and $1672 \mathrm{~cm}^{-1}$ [9] appear at 1609 and $1716 \mathrm{~cm}^{-1}$, whereas the component which corresponds to the $\mathrm{C}=\mathrm{O}$ stretching mode at $1630 \mathrm{~cm}^{-1}$ originating from non-self-associated amide molecules in direct interaction with the water [11] appears at $1670 \mathrm{~cm}^{-1}$. For the observed increase in the frequency of all the three components, we consider that the $\mathrm{H}_{3} \mathrm{PO}_{4}$ molecules produce an extensive breakdown of hydrogen-bonding in the selfassociated amide and in the hydrogen-bonded amide-water species. Similarly to what has been observed with lithium perchlorate, on adding $\mathrm{H}_{3} \mathrm{PO}_{4}$ to a polyacrylamide hydrogel matrix, it is shown that the amide groups become more accessible and the hydrogel flexibility would increase [9].

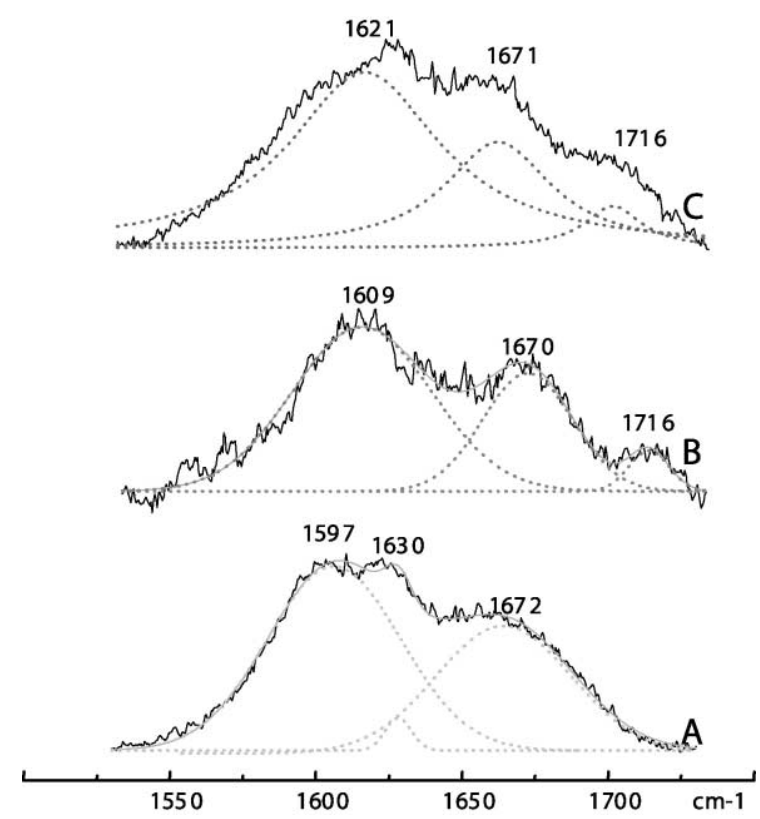

Fig. 2. The Raman spectra of $\mathrm{C}=\mathrm{O}$ stretching vibration, at $295 \mathrm{~K}$, for: (A) polyacrylamide hydrogel; (B) phosphoric acid/polyacrylamide 2:1 mole ratio hydrogel; (C) 2:1:2 phosphoric acid/polyacrylamide/LiCl hydrogel. 
FT-IR studies by Stevens et al. [1] on polyacrylamide-phosphoric acid hydrogels have concluded from the dependency of the water content the presence of various phosphate groups, e.g. as $\mathrm{HPO}_{4}^{2-}$ or $\mathrm{H}_{2} \mathrm{PO}_{4}^{-}$. This presence would imply that the transport of protons may occur via a Grotthuss-type mechanism which involves an exchange of protons between a Bronsted-type acid $\left(\mathrm{H}_{2} \mathrm{PO}_{4}^{-}\right)$and a Bronsted-type base $\left(\mathrm{HPO}_{4}^{2-}\right)$. However, because of the overlapping of most vibrational modes of the phosphoric species with the polyacrylamide vibrational modes in the $1000-1800 \mathrm{~cm}^{-1}$ region, we cannot make any conclusions on this possibility from our measured spectra.

From the polyacrylamide matrix to the $1: 2$ polyacrylamide-phosphoric acid hydrogel network, there are no significant differences either in the polymeric $\mathrm{C}-\mathrm{H}$ stretching modes $\left(2800-3000 \mathrm{~cm}^{-1}\right)$ or in the $\mathrm{N}-\mathrm{H}$ (from acrylamide) and $\mathrm{O}-\mathrm{H}$ (from water and phosphoric acid) stretching modes (3000-3700 $\mathrm{cm}^{-1}$ ).

\subsection{Cation-water interactions in polyacrylamide- phosphoric acid networks}

In Fig. 3, we show the spectra in the water $\mathrm{O}-\mathrm{H}$ stretching region and, in Fig. 4, the observed frequency changes for 1:2 molar ratio polyacrylamidephosphoric acid networks containing lithium, calcium and magnesium chlorides in the proportion of 1 mol of phosphoric acid for 1 or $2 \mathrm{~mol}$ of the chloride.

The general spectroscopic features of $\mathrm{O}-\mathrm{H}$ stretching vibration in pure liquid water are wellknown. Standard curve resolution for the complex band envelope of water gives a weak band at $\sim 3630$ $\mathrm{cm}^{-1}$ assigned to the $v_{3}\left(\mathrm{~b}_{1}\right)$ water $\mathrm{O}-\mathrm{H}$ asymmetric stretching mode, a band at $\sim 3450 \mathrm{~cm}^{-1}$ assigned to the $v_{1}\left(\mathrm{a}_{1}\right)$ water $\mathrm{O}-\mathrm{H}$ symmetric stretching mode; and a band at $\sim 3225 \mathrm{~cm}^{-1}$ corresponding to Fermi resonance of the overtone of the $\mathrm{O}-\mathrm{H}$ bending frequency with the symmetric stretching band at about $3450 \mathrm{~cm}^{-1}$ [12-14].

Based on these assignments, we know that there is a significant decrease in the frequency of the water $\mathrm{O}-\mathrm{H}$ stretching modes in the 1:2 molar ratio polyacrylamide-phosphoric acid hydrogel: $3539 \mathrm{~cm}^{-1}$

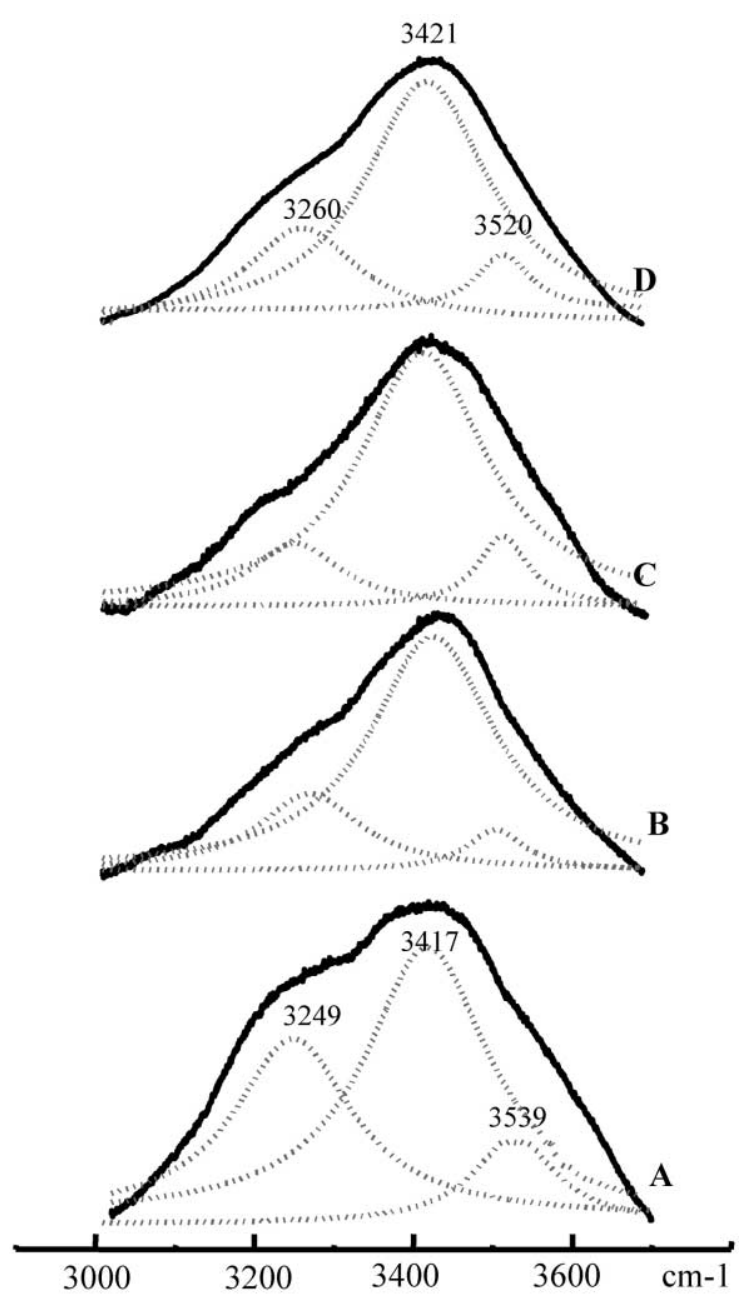

Fig. 3. The Raman spectra of water $\mathrm{O}-\mathrm{H}$ stretching vibration for phosphoric acid/polyacrylamide 2:1 mole ratio hydrogel (A) containing lithium, magnesium and calcium chlorides with molar ratios, relative to the phosphoric acid: (B) lithium chloride $1: 1$; (C) magnesium chloride 1:1; (D) calcium chloride 1:1.

for the $v_{3}\left(\mathrm{~b}_{1}\right)$ asymmetric stretching mode and 3417 $\mathrm{cm}^{-1}$ for the $v_{1}\left(\mathrm{a}_{1}\right)$ water symmetric stretching mode. On the other hand, the frequency of the overtone due to the $\mathrm{O}-\mathrm{H}$ bending and the symmetric stretching mode increases more than $20 \mathrm{~cm}^{-1}$ (from 3225 to $3249 \mathrm{~cm}^{-1}$ ). The similarity of these observed changes with the similar changes observed for the same bands, on going from gas to liquid water due to the hydrogen-bonding leads us to suggest that the polyacrylamide-phosphoric acid network behaves 


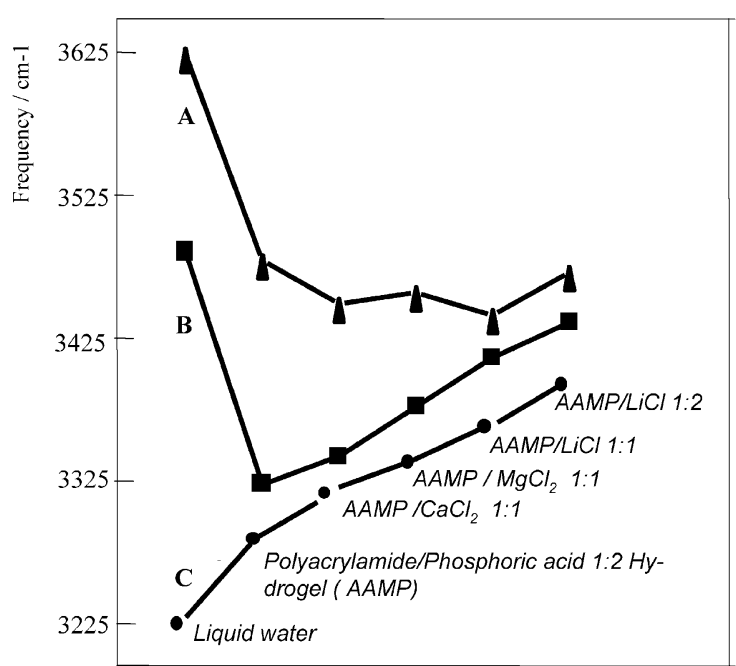

Fig. 4. Frequency changes in the water $\mathrm{O}-\mathrm{H}$ stretching vibration region: (A) the $\mathrm{O}-\mathrm{H}$ asymmetric stretching; (B) the $\mathrm{O}-\mathrm{H}$ symmetric stretching; (C) the Fermi resonance of the overtone of the $\mathrm{O}-\mathrm{H}$ bending with the $\mathrm{O}-\mathrm{H}$ symmetric stretching.

like structure-makers promoting a larger association in the 'bulk' liquid water molecules.

The frequency shift of the $\mathrm{O}-\mathrm{H}$ stretching modes to the lower side on going from pure liquid water to the 1:2 polyacrylamide-phosphoric acid hydrogel, shown in Fig. 4, means that the hydrogel network increases the extent of the water-to-water hydrogen bonding. On the other hand, the shift to higher frequencies of the water $\mathrm{O}-\mathrm{H}$ asymmetric stretching mode on the charge compensating exchangeable cations due to the added chloride salts seems to show that the coordination of the cations with the water oxygens in the polyacrylamide-phosphoric acid hydrogel does not favour the same water-to-water hydrogen bonding. The sequence of this shift to higher frequencies upon the cation nature of the added chloride may mean that the water molecules directly coordinated to the $\mathrm{Ca}^{2+}$ and $\mathrm{Mg}^{2+}$ divalent ions are more strongly polarized than the water molecules coordinated to the lithium ions. The strength of the water-to-water hydrogen bonds clearly corresponds to the increase of the polarising power of the cation.

The observed changes for the water $\mathrm{O}-\mathrm{H}$ stretching modes in the studied polyacrylamide/phosphoric acid/chloride salts hydrogels networks can be understood from the cation coordination with the oxygen atoms of the hydrogen bonded water-to-water molecules. We consider that the main effects of the salt additives in these hydrogels networks lie upon the water molecules in the gel rather than upon the polyacrylamide chains or the phosphoric acid groups attached to them. This consideration may be supported from the unchanged spectral features due to the internal vibrations of these groups with the additives as shown in Fig. 5 which confirm the predominant and effective molecular interactions.

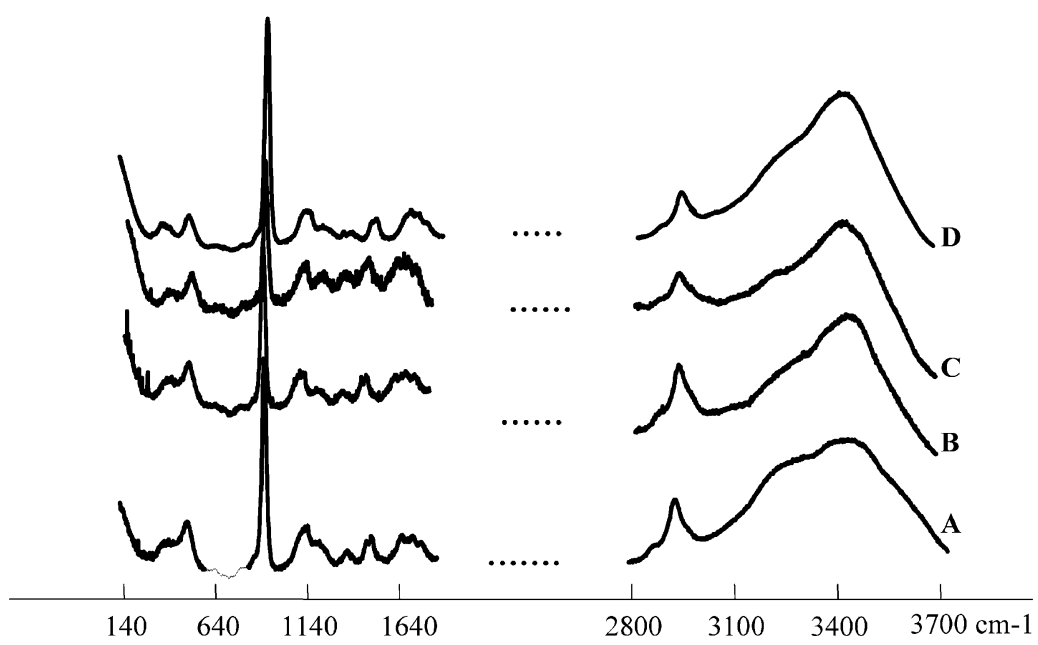

Fig. 5. Raman spectra, at $295 \mathrm{~K}$, for: (A) phosphoric acid/polyacrylamide 2:1 mole ratio hydrogel; (B-D) Phosphoric acid/polyacrylamide 2:1 mole ratio hydrogel doped with $\mathrm{LiCl}, \mathrm{CaCl}_{2}$ and $\mathrm{MgCl}_{2} 1: 1$ phosphoric acid mole ratio, respectively. 


\section{Acknowledgements}

This work had the financial support of Fundação para a Ciência e para a Tecnologia in the Department of Chemistry of the University of Coimbra which the authors greatly acknowledge.

\section{References}

[1] J.R. Stevens, W. Wieczorek, D. Raducha, K.R. Jeffrey, Solid State Ionics 97 (1997) 347, and references therein.

[2] W. Wieczorek, Z. Florjanczyk, J.R. Stevens, Electrochim. Acta 40 (1995) 2327.

[3] W. Wieczorek, A. Zalewska, D. Raducha, Z. Florjanczyk, J.R. Stevens, J. Phys. Chem. B 102 (1998) 352.
[4] Z. Florjanczyk, W. Krawiec, D. Greszta, W. Wieczorek, M. Siekierski, Bull. Electrochem. 8 (1992) 524.

[5] W. Wieczorek, K. Such, H. Wycislik, J. Plocharski, Solid State Ionics 36 (1989) 255.

[6] J.R. McCallum, A.S. Tomlin, C.A. Vincent, Eur. J. 22 (1986) 787

[7] L.C. Thomas, R.A. Chittenden, Spectrochim. Acta 20 (1964) 489.

[8] S. Mohan, R. Murugan, Arabian J. Sci. Eng. 22 (1997) 155.

[9] A.M. Amorim da Costa, A.M. Amado, Polymer 41 (2000) 5361.

[10] G. Fogarasi, A. Balazs, J. Mol. Struct. 133 (1985) 105.

[11] N. Tanaka, K. Ito, H. Kitano, Macromolecules 27 (1994) 540.

[12] G.E. Walrafen, J. Chem. Phys. 36 (1962) 1035.

[13] W.R. Busins, D.F. Hornig, J. Phys. Chem. 65 (1961) 284.

[14] J.R. Scherer, The vibrational spectroscopy of water, in: R.J. Clark, R.E. Hester (Eds.), Adv. Infrared Raman Spectrosc., vol. 5, Heyden, London, 1978, pp. 149-216. 